occurrence of occupational accidents was determined using multivariate logistic regression analyses. Multivariate analyses were adjusted for age, gender, marital status, children (yes/no), job title, multiple employers (yes/no) and the number of flights flown during 2005-2008.

Results In 2009, 289 accidents were reported. Multivariate analyses showed that the number of short-haul flights during 20052008 was positively associated with the occurrence of occupational accidents in $2009(\beta=0.004 ; p=0.012)$. Cumulative exposure to long-haul flights during 2005-2008 proved to be negatively associated with occupational accidents in 2009 ( $\beta=$ $-0.006 ; p=0.011$ ).

Conclusions The results of this study show that cumulative exposure to short-haul flights is associated with an increased risk for occupational accidents among cabin crew. This increased risk may be caused by the specific characteristics of short-haul flights, such as frequent customer service and a high time pressure. Future research should focus on the effects of alternation between different flight schedules and the influence of specific roster characteristics of short-haul schedules.

\section{EFFECTS OF EXPOSURE REDUCTION ON OCCUPATIONAL ALLERGIC DERMATITIS: COMPARISON OF PREVENTION EFFECTIVENESS OF NATIONAL ACTIONS IN UK AND FRANCE}

${ }^{1}$ L B C Bensefa-Colas, ${ }^{2}$ Telle-Lamberton, ${ }^{2}$ Faye, ${ }^{3}$ Luc, ${ }^{2}$ Lasfargues, ${ }^{4}$ Paris, ${ }^{5}$ Agius, ${ }^{5}$ Stocks. ${ }^{1}$ APHP, Paris Descartes University, Paris, France, ${ }^{2}$ ANSES, Paris, France; ${ }^{3}$ INSERMU964, Vandoeuvre-Lès-Nancy, France; ${ }^{4}$ Occupational Diseases Department, University Hospital, INSSERMU964, Vandoeuvre-Lès-Nancy, France; ${ }^{5}$ Centre for occupational and environmental health, University of Manchester, Manchester, United Kingdom

\subsection{6/oemed-2013-101717.405}

Objective Here we present data comparing trends in surveillance of occupational diseases in France and the UK for allergic contact dermatitis attributed to chromate in cement (CDCr) and contact urticaria attributed to latex (UL) within the context of changes in legislation or of national actions to reduce exposure to allergens at these two countries workplace.

Methods The time period for analysis was selected to coincide with the introduction of legislation or compensation in the UK and France (2005 for cement and 2004 for latex). Using the data of two nationwide occupational diseases reporting networks in UK and France (THOR and RNV3P respectively), we analysed the temporal trends of CDCr and of UL over the period 1998-2009. We calculated reporting odd ratio (ROR) with reference to period preceding the change of legislation for the causal agent (cement or latex) relative to time period after intervention, both for British and French data. The ROR compares the ratio of incident cases attributed to one specific allergen relative to all other causal agents, before and after the change in legislation.

Results During 2006-2009 relative to reference period, there was a significant decline of $\mathrm{CDCr}$ in UK construction sector $(\mathrm{ROR}=0.37 ; 95 \%$ IC: $0.18-0.76)$ and a decline of french CD to cement $(\mathrm{ROR}=0.66 ; 95 \%$ IC: $0.0 .35-1.22)$. Concerning $\mathrm{UL}$, there was a decrease in UK healthcare workers $(\mathrm{ROR}=$ 0.84; 95\% IC: 0.57 - 1.25) in 2003-2007 period compared to $1998-2002$ and a decrease in french cases $(\mathrm{ROR}=0.73 ; 95 \%$ IC: 0.43 - 1.24).

Conclusion Through two distinct networks in UK and France, significant decline of $\mathrm{CD}$ attributed to chromate in cement and latex in gloves were observed following actions to reduce occupational exposure. These features are consistent with strong effectiveness of European preventive actions in these two countries.

\section{Session: 35. Occupational respiratory health}

\section{JAPANESE INDIUM COHORT STUDY: FIVE-YEAR FOLLOW-UP}

${ }^{1} \mathrm{M}$ N Nakano, ${ }^{2} \mathrm{~A}$ Tanaka, ${ }^{2} \mathrm{M}$ Hirata, ${ }^{1} \mathrm{~N}$ Yoshioka, ${ }^{1} \mathrm{~K}$ Omae. ${ }^{1}$ School of Medicine, Keio University, Tokyo, Japan; ${ }^{2}$ Graduate School of Medical Sciences, Kyushu University, Fukuoka, Japan

\subsection{6/oemed-2013-101717.406}

Background Since the demand for manufacturing flat panel display expands, we are ongoing an indium cohort study and followed up 310 indium exposure workers for 5 years to assess the changes on the lung effects.

Methods We conducted a baseline epidemiologic survey in 14 indium related plants between 2003 and 2006. A 5-year followup study was conducted in 11 of these 14 initial plants between 2008 and 2011. Follow-up rate is $57.2 \%$ (310/542). Indium concentration in serum (In-S) was determined as an exposure parameter, and effects on the lungs were examined. Some potential confounders were also checked. All participates were classified into three categories, non-, current, and former exposure, according to occupational history of indium exposure.

Results During the follow-up, intensive efforts for improvement of working environments were made in all 11 factories. In currently and formerly exposed workers, mean In-S and geometric means of interstitial biomarkers, KL-6 and SP-D, were significantly lower at follow-up than in the baseline study. In currently and formerly exposed workers, prevalence decreased by approximately $30 \%$ for KL-6 abnormalities but did not significantly decrease at all for $\mathrm{FEV}_{1.0} / \mathrm{FVC}, \% \mathrm{FVC}$, or $\% \mathrm{FEV}_{1.0}$ abnormalities. In the formerly exposed group, chest high-resolution computed tomography (HRCT) showed high prevalence of interlobular septal thickening and emphysematous change (15.1\% and $26.7 \%$, respectively). Aggravation of interstitial change on HRCT was not affected by In-S level, whereas that of emphysematous change progressed in a dose-response manner, even after adjustment for smoking and age. Workers with $\geq \operatorname{In}-\mathrm{S} 20 \mu \mathrm{g} / \mathrm{L}$ had a greater risk of aggravation of emphysematous findings (AOR: 13.31; 95\% CI: 3.29-53.89). During the 5-year-followup, two non-exposed worker at the baseline were diagnosed as lung and stomach cancers, and one currently exposed worker was diagnosed as renal cancer.

Conclusion Emphysematous changes might cause more chronic and longer-term lung effects than interstitial pneumonia.

\section{CHRONIC-OBSTRUCTIVE PULMONARY DISEASE (COPD) CAUSED BY INHALATION OF INORGANIC DUST AT THE WORKPLACE - SYSTEMATIC REVIEW AND METAANALYSIS}

${ }^{1} \mathrm{M}$ S Brueske, ${ }^{1}$ Thiering, ${ }^{1}$ Heinrich, 'Wichmann, ${ }^{2}$ Huster, ${ }^{2}$ Nowak. ${ }^{1}$ Helmholtz Zentrum München, Neuherberg, Germany, ${ }^{2}$ Ludwig-Maximilians-Universität, München, Germany

\subsection{6/oemed-2013-101717.407}

Objective A systematic review of the scientific medical literature on COPD and the inhalative exposure to inorganic dust and a metaanalysis. 
Methods The search was done in Medline/EMBASE. The quality of selected papers was evaluated on the basis of the NewcastleOttawa Scale and the recommendations of the Cochrane Collaboration. Metaanalysis of the results of pulmonary function tests were evaluated separately for bio-persistent granular dust (BGD) and silica containing dust by random-effects models.

Results In cross-sectional analyses subjects occupationally exposed to BGD had on average a $160 \mathrm{ml}$ lower FEV1 and the predicted value for FEV1 was decreased by $5.7 \%$. The ratio of FEV1/FVC was also significantly decreased. Related to a cumulative BGS- exposure of $1 \mathrm{mg} / \mathrm{m}^{3}$.year FEV1 was decreased by $1.6 \mathrm{ml}$. The Odds Ratio for obstructive pulmonary test results (FEV1/FVC <70\%) was 1.07 per $1 \mathrm{mg} / \mathrm{m}^{3}$ BGD at the workplace. Longitudinal analyses showed a yearly decline of FEV1 of $6.3 \mathrm{ml}$ in BGD-exposed and of $11.8 \mathrm{ml}$ in silica exposed persons compared to non/low-exposed persons in addition to age-dependent decline. A study of uranium miners (WISMUT Company) in Germany showed that the cumulative exposure to $1 \mathrm{mg} / \mathrm{m}^{3}$ pure respirable silica dust decreased the ratio of FEV1/FVC by $2.75 \%$. The risk of COPD stage I increased (OR 1.81 pro $1 " \mathrm{mg} / \mathrm{m}^{3} \cdot$ year) related to the exposure of pure respirable silica dust. In relation to COPD stage II + the cumulative doubling dose was $2 \mathrm{mg} / \mathrm{m}^{3}$.year pure respirable silica dust. All cited results were statistically significant $(\mathrm{p}<$ 0.01 to $\mathrm{p}<0.001)$. Funnel plots did not indicate major publication bias.

Conclusion Review and metaanalysis showed a statistically positive association between the inhalative exposure to BGD at the workplace and a decreased FEV1 in cross-sectional as well as in longitudinal analyses.

Funded by the Federal Ministry of Labour and Social Affairs (“Bundesministerium für Arbeit und Soziales”).

\section{MEASURES OF METALWORKING FLUID EXPOSURE AND ITS RELATIONSHIP TO RESPIRATORY HEALTH IN MACHINISTS}

${ }^{1}$ E Peters, ${ }^{2}$ Arrandale, ${ }^{1}$ Teschke, ${ }^{3}$ Turner, ${ }^{4}$ Chambers, ${ }^{5}$ McLean, ${ }^{1}$ Karlen, ${ }^{1}$ Kennedy. ${ }^{1}$ University of British Columbia, Vancouver, Canada; ${ }^{2}$ Division of Preventive Medicine, University of Alberta, Edmonton, Canada; ${ }^{3}$ Faculty of Medicine, McGill University, Montreal, Canada; ${ }^{4}$ Faculty of Medicine, University of Ottawa, Ottawa, Canada; ${ }^{5}$ Faculty of Health Sciences, Simon Fraser University, Burnaby, Canada

\subsection{6/oemed-2013-101717.408}

Objectives Our objective was to investigate the relationship between metalworking fluid (MWF) exposure and subsequent respiratory health among machinists in an inception cohort of apprentices.

Methods All first-year machinist apprentices at a provincial trade school were eligible to enrol (visit 1: 1988-1990). Subjects were retested during their $3^{\text {rd }}$ year (visit 2: 1990-1992) and again 16 years after baseline (visit 3: 2004-2006). Interviewers collected information on respiratory symptoms and personal, work and exposure histories. Questionnaire responses were used to model average MWF exposure intensity for each job. Duration and cumulative MWF exposure were subsequently estimated. Pulmonary function and bronchial hyper-responsiveness (BHR) were also measured. Multiple linear regression models were constructed to investigate the relationship between MWF exposure and both $\%$ predicted forced expiratory volume in 1 second (\% P-FEV1) and BHR at visit 3.

Results At visit 1, 99 machinist apprentices were enrolled. At visit 3, 77 (77\%) machinists participated; all were men (mean age 40.6 years). Machinists who had left the trade tended to have lower \% P-FEV1 at visit $3(\mathrm{p}=0.10)$ than current machinists and were more likely to have BHR $(26 \%$ vs. $9 \%, p=0.04)$. In regression models, leaving the machining trade was associated with lower \% P - FEV1 at visit 3 $(\mathrm{p}=0.005)$. Early MWF exposure was associated with lower $\%$ P - FEV1 at visit $3(\mathrm{p}=0.02)$. In a separate model, current MWF exposure was associated with increased BHR ( $p$ $=0.05)$.

Conclusions Exposure to MWF was associated with \% P-FEV1 and BHR at the 16 year follow-up. Early MWF exposure was associated with decreased \% P-FEV1 and current exposure was associated with increased BHR. Machinists who left the trade had decreased \% P-FEV1 and increased BHR at 16 year followup, demonstrating the strength of the healthy worker effect in this population and the need to account for this effect in studies of work-related respiratory disease.

\section{THE USE OF AN AUTOPSY COMPENSATION DATABASE FOR SURVEILLANCE OF PULMONARY TUBERCULOSIS IN THE SOUTH AFRICAN MINING INDUSTRY: 1975-2011}

${ }^{1} \mathrm{~N}$ Nldovu, ${ }^{2}$ Park, ${ }^{3}$ Murray. ' Naitonal Institute for Occupational Health, Johannesburg, South Africa; '2School of Public Health, University of Michigan, Ann Arbor, MI, United States of America; ${ }^{3}$ National Institute for Occupational Health, Johannesburg, South Africa

\subsection{6/oemed-2013-101717.409}

Introduction Pulmonary tuberculosis (PTB) is a major public health problem in South Africa and gold miners have the highest incidence of tuberculosis in the world. However, there is no systematic surveillance of occupational lung diseases in the mining industry. South African law allows for autopsy examination for the diagnosis of occupational lung diseases, including PTB, regardless of the cause of death and with consent from the next of kin. Since 1975, the autopsy findings have been stored on a computerised database (PATHAUT). We estimated temporal trends in the relative proportions of PTB and evaluated their use for disease surveillance.

Methods We extracted data from PATHAUT on all autopsies from 1975 to 2011. The data were stratified by population group because of differences in the patterns of exposure, employment and autopsy referral. Long-term PTB trends and potential determinants of these trends such as age, gold mining service, duration of employment and year of death, were evaluated using linear regression.

Results The crude proportions of PTB were higher in black than white miners. The proportion of black miners with PTB increased from 0.03 in 1975 to 0.3 in 2011 and from 0.01 to 0.06 in white miners. Most of the miners with PTB (79.3\%) had been exposed to silica dust in the gold mining industry. The increasing PTB trend was primarily due to year of death, increasing age and employment duration in the gold mining industry.

Conclusions The high and increasing trend of PTB in the South African mining industry is of great concern. Our findings will be discussed in the context of high HIV prevalence and silica dust levels in the mining industry. Initiatives to control the epidemic are underway and the autopsy database provides data that can be used for the surveillance of PTB and monitoring of preventative interventions. 